\title{
Synergy of CO/[CII]/Ly $\alpha$ Line Intensity Mapping with the SKA
}

Tzu-Ching Chang*1, Yan Gong ${ }^{2}$, Mario Santos ${ }^{3,4,5}$, Marta Silva ${ }^{4}$, James Aguirre ${ }^{6}$, Olivier Doré $^{7,8}$, Jonathan Pritchard ${ }^{9}$, on behalf of the EoR/CD-SWG

${ }^{1}$ Academia Sinica Institute of Astronomy and Astrophysics, P.O. Box 23-141, Taipei, 10617 Taiwan

${ }^{2}$ Department of Physics \& Astronomy, University of California, Irvine, CA 92697, USA

${ }^{3}$ Department of Physics, University of Western Cape, Cape Town 7535, South Africa

${ }^{4}$ CENTRA, Instituto Superior T İAecnico, Technical University of Lisbon, Lisboa 1049-001, Portugal

${ }^{5}$ SKA SA, 3rd Floor, The Park, Park Road, Pinelands, 7405, South Africa

${ }^{6}$ Department of Physics \& Astronomy, University of Pennsylva-nia, 209 South 33rd Street, Philadelphia, PA 19104, USA

${ }^{7}$ NASA Jet Propulsion Laboratory, California Institute of Technology, 4800 Oak Grove Drive, MS 169-215, Pasadena, CA, 91109, U.S.A.

${ }^{8}$ California Institute of Technology, MC 249-17, Pasadena, California, 91125 U.S.A.

${ }^{9}$ Department of Physics, Blackett Laboratory, Imperial College, London SW7 2AZ, UK

E-mail: tcchang@asiaa.sinica.edu.tw

This subchapter describes the science enabled by cross-correlations of the SKA1-LOW 21-cm EoR surveys with other line mapping programs. In particular, we identify and investigate potential synergies with planned programs, such as the line intensity mapping of redshifted $\mathrm{CO}$ rotational lines, $[\mathrm{CII}]$ and Ly- $\alpha$ emissions during reionization. We briefly describe how these tracers of the star-formation rate at $z \sim 8$ can be modeled jointly before forecasting their autoand cross-power spectra measurements with the nominal $21 \mathrm{~cm}$ EoR survey. After discussing the measurement prospect, we discuss how reionization parameters can be better constrained using these new measurements.

Advancing Astrophysics with the Square Kilometre Array

June 8-13, 2014

Giardini Naxos, Italy

\footnotetext{
*Speaker.
} 


\section{Introduction}

While the distribution of neutral hydrogen mapped by SKA1-LOW provides an excellent and unique view of the reionization process over a large range of redshifts, detecting the sources responsible for reionization directly sheds light on the crucial stage of galaxy formation and complements our understanding of EoR. Extremely deep imaging with the Hubble Space Telescope (HST) has begun to probe the very bright end of the UV luminosity functions at $\mathrm{z}>6$ (Bouwens et al. 2014; Robertson et al. 2013), with improvements expected with the James Webb Space Telescope (JWST). In the sub-mm, the Atacama Large Millimeter Array (ALMA) has detected individual high redshift, luminous objects known from existing surveys (e.g., Ouchi et al. (2013)). However, observations that are aimed at detecting individual galaxies at $\mathrm{z}>6$ are difficult and time consuming, and neither of these space-borne facilities nor ALMA is expected to resolve the bulk of low luminosity sources responsible for reionization (Salvaterra et al. 2011). Approaches which can access the entire luminosity function of reionizing sources are needed.

Line Intensity Mapping has emerged as a promising technique that is sensitive to the integrated light produced by faint galaxies: instead of resolving individual sources, one measures on larger spatial scales the collective emission from an ensemble of sources, while retaining the spectral-thus redshift-information. This allows efficient redshift surveys that probe the integrated luminosity function of sources and provide three-dimensional information to study star formation activities during EoR.

A few spectral lines are currently being considered as promising tracers for high-redshift star formation activities in the intensity mapping regime. Among them, the most promising ones are the rotational transitions from carbon monoxide (CO) (Righi et al. 2008; Visbal \& Loeb 2010; Carilli 2011; Gong et al. 2011; Lidz et al. 2011; Pullen et al. 2013; Breysse et al. 2014), the $158 \mu \mathrm{m}$ emission from singly ionized carbon ([CII]) (Gong et al. 2012; Uzgil et al. 2014; Silva et al. 2014), and the Lyman- $\alpha$ transition line from hydrogen (Silva et al. 2013; Pullen et al. 2014). Such largescale surveys will not only reveal early star formation history but also measure the clustering of ionizing sources. These line intensity maps mark the three-dimensional distribution of ionized regions and probe different gas phases that complements the $21 \mathrm{~cm}$ EoR surveys which trace neutral hydrogen. Together, they draw a complete view of the reionization process in the high-redshift Universe. In addition, on scales larger than the ionized regions, these line tracers anti-correlate with the $21 \mathrm{~cm}$ emission. By measuring the shape of the cross-power spectrum of the two surveys, one can determine the characteristic scale of ionized regions, by marking the scale where the crosscorrelation becomes negative, and be able to constrain statistically the characteristic size scale of ionized regions as a function of redshifts (Righi et al. 2008; Lidz et al. 2009).

Below we discuss each of these tracers in detail, and present forecasts on the measurements of power spectra and cross-correlation signals with the SKA1-LOW $21 \mathrm{~cm}$ EoR survey. Due to the short emission wavelengths, it is not possible to observe [CII] and Ly $\alpha$ with the SKA, thus we present the results assuming other future surveys. For CO, however, the proposed highestfrequency band of SKA1-MID can potentially cover the redshifted $\mathrm{CO}(1-0)$ transition at $z>7.3$. We discuss such possibilities in the next sections. 


\section{CO Intensity Mapping}

The $\mathrm{CO}(1-0)$ rotational line has a rest frequency of $115 \mathrm{GHz}$. The proposed highest frequency band of SKA1-MID, 4.6-13.8GHz, will have a chance of capturing the redshifted $\mathrm{CO}(1-0)$ at $z>7.3$. For intensity mapping purposes, where we aim to measure the large-scale distribution of redshifted $\mathrm{CO}$ at low-angular resolution, only the inner few kilometer core of SKA1-MID will be relevant. The uncertainty in the theoretical modelling of $\mathrm{CO}$ brightness temperature at high redshifts is still large, but most predict the amplitude to be about $10^{-6} \mathrm{~K}$ or smaller on quasi-linear scales. Large collecting area and high sensitivity are required, and a densely packed antenna configuration at the central core is desired, much like the requirement for SKA1-LOW for the $21 \mathrm{~cm}$ EoR survey. Currently, however, the designed filling factor of SKA1-MID at the core is relatively small, only about $10^{-5}$ in the inner $1 \mathrm{~km}$ core in diameter, making the prospect of detecting redshifted $\mathrm{CO}$ very challenging.

Here we instead use SKA1-MID as a collection of single dishes, where each antenna records the total power while the cross products between antenna pairs are ignored. We note that performing the CO survey in the single dish mode lifts the compact antenna configuration requirement and enables a survey in the intensity mapping regime, but imposes constraints on the stability of the auto-correlation spectrum which would not be present if it were done interferometrically. We consider a narrow redshift range at $z=8 \pm 0.5$, the lowest possible redshift allowed by SKA1-MID which should be more CO-rich. The specification is listed in Table 1, which also specifies the assumed SKA1-LOW $21 \mathrm{~cm}$ survey parameters. Note that the assumed survey areas for CO autopower spectrum and CO-HI cross-power spectrum calculations are chosen to be 0.1 and $10 \mathrm{deg}^{2}$, respectively, in order to optimize the signal-to-noise ratio in each case.

The amplitude of predicted $\mathrm{CO}$ brightness temperature fluctuations is rather uncertain, differing by orders of magnitude at $z=8$ from model to model. This is currently one of the most challenging aspects for $\mathrm{CO}$ intensity mapping work. Figure 1 shows the forecasted $\mathrm{CO}$ power spectrum and the $\mathrm{COx} 21 \mathrm{~cm}$ cross-power spectrum measurements with the survey parameters listed in Table 1. Here we demonstrate the model uncertainties by plotting three of them: the middle solid curve with error bars is based on the models in Righi et al. (2008), while the upper dashed curve is based on Lidz et al. (2011) and lower dashed curve from Gong et al. (2011). The error bars include contributions from thermal noise and cosmic variance. The predicted total signal-to-noise ratio (SNR), summed over all accessible scales, of the upper, central and lower power spectra with SKA1-MID in single dish mode are 20.7, 4.0 and 0.1 ; for the $\mathrm{CO}$ and $21 \mathrm{~cm}$ cross-power spectra with SKA1-MID and SKA1-LOW, the corresponding SNRs are 26.1, 6.0 and 0.7. The CO(1-0) signals and the cross power spectrum between $21 \mathrm{~cm}$ and $\mathrm{CO}(1-0)$ emissions would be detectable at statistically significant level in more optimistic scenarios. The CO model uncertainties, however, exceed the measurement errors, thus making it difficult to plan a survey. On the other hand, a detection of $\mathrm{CO}$ power spectrum can be very discriminating against models and guide our theoretical understandings. Observational efforts for mapping $\mathrm{CO}$ at modest redshifts $(z=2-3)$ with existing instruments have only reached initial results, probing the high-end of CO luminosity functions (Decarli et al. 2014; Walter et al. 2014; Hodge et al. 2014). It is essential to improve our theoretical modelling and advance observational measurements of $\mathrm{CO}$ brightness temperature fluctuations across redshifts, which is currently an active area of research. In addition, one may 
need to worry about foreground contaminations from synchrotron and free-free radiations coming from the Galaxy and other extragalactic sources. The severity depends on the observing frequencies (or the redshifted $\mathrm{CO}$ rotational lines of interest) and the $\mathrm{CO}$ signal strengths. However, these foregrounds are expected to be spectrally smooth and can be separated from the line emissions (Angelakis et al. 2009; Keating et al. 2014). At a few tens of gigahertz frequency range, spinning dust may also be a potential contamination, although its spectrum and strength are not well known (Ali-Haïmoud 2013). It will likely require a dedicated experiment with both a larger field-of-view and greater surface brightness sensitivity to make a strong $\mathrm{CO}$ detection, or a significantly more compact SKA-MID core than currently envisaged.
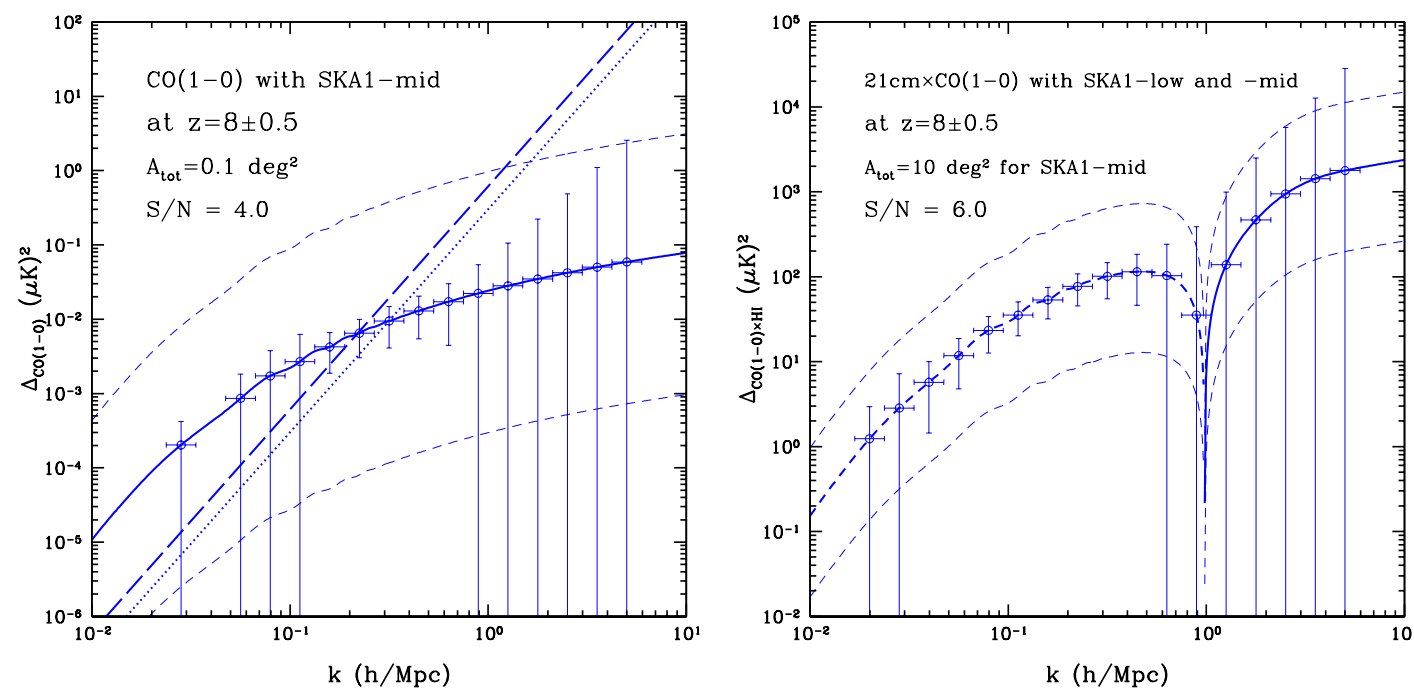

Figure 1: Left: SKA1-MID CO power spectrum at $z=8$. Right: SKA1-LOW $21 \mathrm{~cm} \times$ SKA1-MID CO at $z=8$. The upper, central, and lower curves indicate predicated $\mathrm{CO}$ signal strengths from three different models (see texts), whose uncertainties are larger than the predicted error bars. The survey areas for CO auto-power spectrum and CO-HI cross-power spectrum calculations are assumed to be 0.1 and $10 \mathrm{deg}^{2}$, respectively, to optimize the signal-to-noise ratios.

\section{CII Intensity Mapping}

Carbon is one of the most abundant elements in the Universe and it becomes singly ionized [CII] with an ionization energy of $11.26 \mathrm{eV}$, less than that of hydrogen. With a splitting of the fine-structure level at $91 \mathrm{~K},[\mathrm{CII}]$ is easily excited resulting in a line emission at $157.7 \mu \mathrm{m}$ through the ${ }^{2} P_{3 / 2} \rightarrow{ }^{2} P_{1 / 2}$ transition. It is well established that the bulk of [CII] emission comes from photodissociation regions (PDRs), and provides a major cooling mechanism for the neutral interstellar medium (ISM). It is generally the brightest emission line in star-forming galaxy spectra and contributes to about $0.1 \%$ to $1 \%$ of the total far-infrared (FIR) luminosity in low redshift galaxies. Since carbon is naturally produced in stars, [CII] emission is expected to be a good tracer of the gas distribution in galaxies. ALMA high-resolution observations have revealed [CII] in high-redshift 
Table 1: Parameters for SKA1-LOW and -MID at $\mathrm{z}=8 \pm 0.5$. For SKA1-MID, single dish observation mode using 254 antennae is assumed. The survey areas for CO auto-power spectrum and CO-HI cross-power spectrum calculations are assumed to be 0.1 and $10 \mathrm{deg}^{2}$, respectively, in order to optimize the signal-tonoise ratios.

\begin{tabular}{l|c|c|c}
\hline \hline & SKA1-LOW & $\begin{array}{c}\text { SKA1-MID } \\
\text { auto/cross }\end{array}$ & unit \\
\hline ant. diameter $D_{\text {ant }}$ & 35 & 15 & $\mathrm{~m}$ \\
survey area $A_{\mathrm{s}}$ & 13 & $0.1 / 10$ & $\mathrm{deg}^{2}$ \\
FoV per ant. & 13 & 0.01 & $\mathrm{deg}^{2}$ \\
effective area per ant. $A_{e}$ & 925 & 170 & $\mathrm{~m}^{2}$ \\
freq. resolution $d v$ & 3.9 & 9.7 & $\mathrm{kHz}$ \\
bandwidth $(\mathrm{z}=8 \pm 0.5) \mathrm{BW}$ & 18 & 1427 & $\mathrm{MHz}$ \\
tot. int. time $t_{\text {int }}$ & 1000 & 10,000 & $\mathrm{hr}$ \\
min. baseline $D_{\min }$ & 30 & - & $\mathrm{m}$ \\
max. baseline $D_{\max }$ & 1 & - & $\mathrm{km}$ \\
uv & 16 & - & \\
uvin & 526 & - & \\
$\mathrm{T}_{\text {sys }}$ & 400 & 25 & $\mathrm{~K}$ \\
effective num. ant. & 433 & 254 & \\
num. density of baselines $n_{\text {base }}$ & 0.8 & - & \\
\hline
\end{tabular}

galaxies, e.g, (Riechers et al. 2014; De Breuck et al. 2014), although no detections have been made for [CII] associated with galaxies at $z>\sim 7$ (González-López et al. 2014; Ota et al. 2014)

Even if the angular resolution to resolve the [CII] emission from individual galaxies at high redshift is not available, the brightness variations of the [CII] line intensity can be used to map the underlying distribution of galaxies and dark matter (Basu et al. 2004; Visbal \& Loeb 2010; Gong et al. 2012).

Here we follow Gong et al. (2012) to calculate the expected CII line fluctuations at $6<z<9$, assuming that $[\mathrm{CII}]$ emission mainly originates in the hot gas in galaxies and that it is proportional to the gas metallicity, based on both analytical arguments and numerical models. Alternatively, [CII] emission can be estimated using observational relations, such as the empirical relation between [CII] luminosity and star formation rate (SFR) in low redshift galaxies from Sargsyan et al. (2012). This has the advantage of including [CII] emission from several media, since [CII] is emitted not only from hot ionized gas but also from cold, mostly neutral gas in PDRs, and also in minor proportion from other regions. The connection between [CII] emission and SFR can be easily understood both in PDRs, where the emission of radiation is proportional to the strength of the far UV radiation field thus to the SFR, and in ionized regions since their size is proportional to the ionization rate and thus to the SFR.

For redshifted [CII] line, the redshift range corresponds to observing frequencies of $\sim 200-$ $300 \mathrm{GHz}$. We assume a future [CII] intensity mapping instrument, based on a grating spectrometer and 20,000 bolometer array detectors for spectral line measurements. The instrument is assumed 
to be on a telescope with a $10-\mathrm{m}$ aperture. The details specifications are listed in Table 2. The forecasted cross-power spectrum of $21 \mathrm{~cm}$ and [CII] surveys at $z=8$ is shown in Figure 2. The red curve is the predicted amplitude of cross-correlation based on [CII] models in Gong et al. (2012), while the green curves indicate the theoretical uncertainties. Note the cross-correlation is negative at $k<\sim 1(\mathrm{~h} / \mathrm{Mpc})$, a signature from the anti-correlation of [CII] and $21 \mathrm{~cm}$ on scales larger than the typical bubble size, as the former traces star formation activities thus the ionized region, while $21 \mathrm{~cm}$ traces the neutral part of the Intergalactic Medium (IGM). A 4.7- $\sigma$ detection is expected with this particular setup. At these frequencies, however, contributions from different CO rotational line emissions coming from different redshifts may confuse the redshifted [CII] emissions. One may apply bright-source masking or template fitting techniques to extract the redshifted [CII] signals. A pilot [CII] intensity mapping experiment, Time-Pilot, is currently underway to map out the redshifted [CII] emissions from high redshifts (Crites et al. 2014).

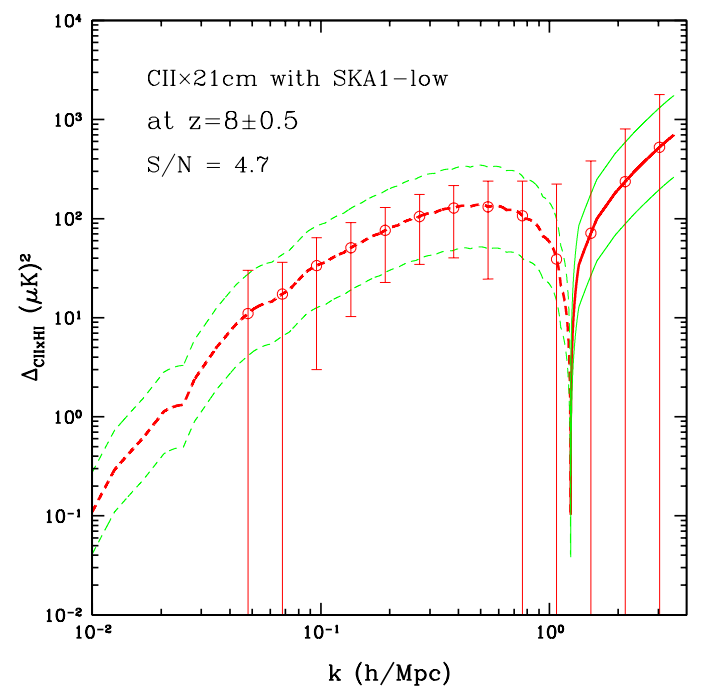

Figure 2: The cross-power spectrum of SKA1-LOW $21 \mathrm{~cm}$ with a potential [CII] line mapping program at $z=8$. The expected signals are plotted in red, while the theoretical uncertainties of the models are indicated by the green curves. The error bars are calculated based on parameters listed in Tables 1 and 2 .

\section{Ly $\alpha$ Intensity Mapping}

Ly $\alpha$ photons have a rest-frame wavelength of $1216 \AA$ and so Ly $\alpha$ emission during the EoR will redshift to the near-infrared regime today, making it potentially detectable by narrow-band infrared detectors. Ly $\alpha$ photons emitted by galaxies are mostly absorbed and reemitted by the neutral hydrogen in the galaxy which causes a scatter of the radiation greatly decreasing the Ly $\alpha$ flux detected by direct observations of Ly $\alpha$ emitters. Therefore, galaxy surveys are not able to fully measure all of the intrinsic Ly $\alpha$ emission. Intensity mapping is however a low resolution technique and so by not attempting to resolve the sources of $\operatorname{Ly} \alpha$ photons we can in principle detect all of the Ly $\alpha$ radiation emitted both from galaxies and from the IGM. 
Table 2: Experimental Parameters for a Possible [CII] Mapping Instrument.

\begin{tabular}{|c|c|}
\hline Aperture diameter $(\mathrm{m})$ & 10 \\
\hline Survey Area $\left(A_{S} ; \operatorname{deg}^{2}\right)$ & 16 \\
\hline Total integration time (hours) & 4000 \\
\hline Free spectral range $\left(B_{v} ; \mathrm{GHz}\right)$ & $185-310$ \\
\hline Freq. resolution $\left(\delta_{v} ; \mathrm{GHz}\right)$ & 0.4 \\
\hline Number of bolometers & 20,000 \\
\hline Number of spectral channels & 312 \\
\hline Number of spatial pixels & 64 \\
\hline Beam size $^{\mathrm{a}}\left(\theta_{\text {beam }} ; \mathrm{FWHM}\right.$, arcmin $)$ & 0.4 \\
\hline Beams per survey area $a^{a}$ & $2.6 \times 10^{5}$ \\
\hline$\sigma_{\text {pix }}:$ Noise per detector sensitivity $(\mathrm{Jy} \sqrt{\mathrm{s}} / \mathrm{sr})$ & $2.5 \times 10^{6}$ \\
\hline$t_{\text {pix }}^{\text {obs }}:$ Integration time per beam ${ }^{\mathrm{a}}$ (hours) & 1.0 \\
\hline$z=8 V_{\mathrm{pix}}(\mathrm{Mpc} / \mathrm{h})^{3}$ & 4.8 \\
\hline$z=8 P_{N}^{\mathrm{CII}}(\mathrm{Jy} / \mathrm{sr})^{2}(\mathrm{Mpc} / \mathrm{h})^{3}$ & $4.3 \times 10^{9}$ \\
\hline
\end{tabular}

${ }^{\text {a }}$ values computed at $238 \mathrm{GHz}$, corresponding to [CII] at $z=7$.

Recent work by Silva et al. (2013); Pullen et al. (2014) describes the process of Ly $\alpha$ emission in the EoR and post-EoR and shows estimates for intensity mapping of the Ly $\alpha$ signal at redshifts $7<z<11$. Ly $\alpha$ emission from galaxies is mainly sourced by stellar ionizing radiation since stars emit photons which ionize neutral hydrogen, which then emits Ly $\alpha$ photons upon recombination, and also because the heating of the gas by stellar UV radiation gives rise to e-HI collisions causing further Ly $\alpha$ emission. During the EoR stellar populations can also source emission in the dense and ionized IGM surrounding the galaxies through e-p recombinations and e-HI collisions. In addition, diffuse Ly $\alpha$ emission is also important. It can originate either from e-p recombinations sourced by $\mathrm{X}$-ray radiation or from stellar continuum photon redshifted into the Ly $\alpha$ line of the IGM.

The intensity of the contributions from galaxies and from the IGM is dependent on several key astrophysical parameters such as: the star formation efficiency, the stellar spectrum, the escape fraction of ionizing photons from galaxies to the IGM, the gas temperature and clumping, the minimum mass of Ly $\alpha$ halos and the ionization state of the IGM. The relative contribution from galaxies and from the IGM to the Ly $\alpha$ photons budget is highly dependent on the ionization history and on the local heating of the IGM and so it is very difficult to estimate.

Observational maps of Ly $\alpha$ emission will be contaminated by extragalactic continuum emission and line foregrounds and also by emission from our galaxy. Continuum contamination can in principle be removed from intensity maps taking into account the smooth evolution of this radiation with frequency compared to the evolution of the Ly $\alpha$ line; however, zodiacal light emitted from our galaxy will bring confusion to the observational maps making it possible to only extract the Ly $\alpha$ power spectra at small scales where zodiacal light is spatially smooth. Foreground lines from lower redshifts, namely the $6563 \AA \mathrm{H} \alpha$, the $5007 \AA$ [OIII] and the $3727 \AA$ [OII] lines will strongly affect Ly $\alpha$ observational maps however their contamination can be removed by masking the contaminated observational pixels as was shown in Gong et al. (2014).

Below we illustrate the cross-correlation signature of Ly $\alpha$ and $21 \mathrm{~cm}$ emissions during EoR, 
Table 3: Experimental Parameters for a Possible Ly $\alpha$ Mapping Instrument.

\begin{tabular}{l|c}
\hline \hline Aperture diameter $(\mathrm{m})$ & 0.2 \\
\hline Survey Area $\left(A_{\mathrm{S}} ; \mathrm{deg}^{2}\right)$ & 13 \\
Total integration time (hours) & 2900 \\
Free spectral range $\left(B_{\lambda} ; \mu \mathrm{m}\right)$ & $0.85-1.1$ \\
Freq. resolution $\left(\lambda / \delta_{\lambda}\right)$ & 220 \\
Number of pixels in 2D array & 72900 \\
FOV per pointing; deg & 0.6 \\
Observational time per pointing (hours) & 129.5 \\
Survey volume $(\mathrm{Mpc} / \mathrm{h})^{3}$ & $8.5 \times 10^{7}$ \\
\hline \hline
\end{tabular}

from an assumed Ly $\alpha$ intensity mapping experiment which consists of an aperture array with the parameters described in table 3 . The parameters of the $21 \mathrm{~cm}$ intensity mapping observation with SKA1-LOW are described in Table 1 for $z=8$, and the assumed frequency dependence of instrument system temperature $T_{s y s}$ and the collecting area $A_{e}$ are as follows: $T_{s y s}=T_{s k y}+T_{\text {rec }}$, where $T_{s k y}=60\left(\frac{300 \mathrm{MHz}}{v}\right)^{2.55} \mathrm{~K}$ is the sky temperature in Kelvin, and $T_{r e c}=0.1 * T_{s k y}+40 \mathrm{~K}$ is the instrument receiver temperature. $A_{e}=925\left(\frac{110}{v}\right)^{2} \mathrm{~m}^{2}$. The Ly $\alpha$ intensity mapping calculation is from Silva et al. (2013). The forecast shows that the $21 \mathrm{~cm}$ and Ly $\alpha$ cross-power spectra can be detected at high SNR values of $(789,442,273,462)$ for $z=(7,8,9,10)$, respectively, and is shown in Figure 3.

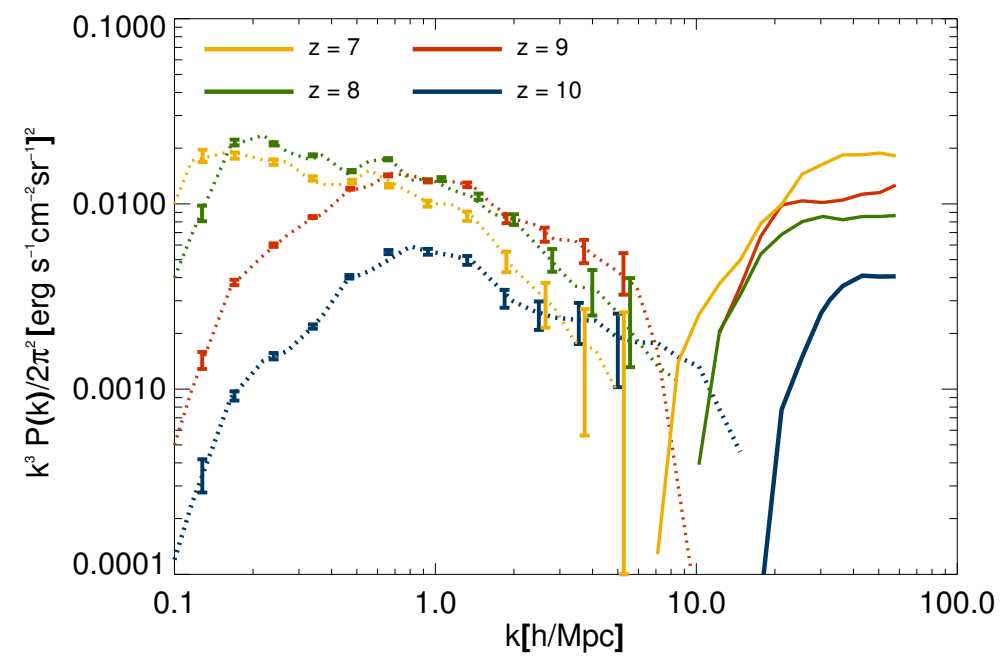

Figure 3: The SKA1-LOW $21 \mathrm{~cm}$ and Ly $\alpha$ cross-power spectra at $z=(7,8,9,10)$. The Ly $\alpha$ models are based on Silva et al. (2013). The cross-power spectra are predicted to be detectable at $>100$ SNR significance level, given the assumed survey parameters 


\section{Discussion}

The strength of the correlation between HI and molecular line emission depends on multiple factors and in particular, the sign of the correlation will depend on whether the emission comes mostly from the galaxies or the IGM. When cross-correlating CO with HI, the cross-correlation signals are expected to be associated with the clustering of galaxies (CO) and the IGM (HI), thus there is a strong and easy to interpret anti-correlation. When cross-correlating [CII] with HI, the bulk of the $[\mathrm{CII}]$ emissions are expected to come from galaxies, with some small contributions from the IGM, and we expect a strong anti-correlation. At the high redshifts of interest $(z>6)$, a small amount of metals may reside in the IGM, however, the [CII] spin temperature is expected to follow the CMB temperature (Gong et al. 2012) thus little [CII] emission from the IGM is expected. For cross-correlations of $\mathrm{HI}$ with $\mathrm{Ly} \alpha$ emissions, the situation is more complicated since the Ly $\alpha$ emission from the IGM can be very high and is very uncertain. Depending on the model considered, the emission from the IGM can even be higher than the emission from galaxies at some redshifts. In this case, $\operatorname{Ly} \alpha$ and $\mathrm{HI}$ would be positively correlated on large scales; on the other hand, if no Ly $\alpha$-HI cross-correlation signals are found, we can place constraints on the Ly $\alpha$ sources of emission.

\section{Summary}

In this chapter we motivated a novel use of SKA1-LOW $21 \mathrm{~cm}$ to probe the epoch of reionization. By cross-correlating the $21 \mathrm{~cm}$ line with other atomic and molecular lines observed in the intensity mapping regime, we can not only validate a potential $21 \mathrm{~cm}$ EoR detection but also learn more about the EoR itself. On the one hand, the $21 \mathrm{~cm}$ line traces neutral regions, the yet to be ionized universe while the other atomic lines trace star formation activities. The combination thus forms a potent, complete and unique picture of the reionization process.

We focus our study on $\mathrm{CO},[\mathrm{CII}]$ and $\mathrm{Ly} \alpha$ transition lines currently identified as the most promising tracers. For CO, we study the possibility of using directly SKA1-MID at appropriate frequencies to map the EoR at z>7.5. For [CII] and Ly $\alpha$, we assume the successful deployment of relevant instruments currently being planned. In all cases, we find that the strength of our detection strongly varies with theoretical model. For example, in the case of $\mathrm{CO}$, according to the particular models considered, we could go from a strong detection (greater than $6 \sigma$ ) to a non-detection. The same holds for CII, and it appears more promising for Ly $\alpha$ to be detected at high SNRs . While this situation might be worrisome, it simply reflects the fact that we are probing a totally new territory for astrophysics. Besides, these line tracers have different sources of astrophysical contamination which are in general much less severe than the ones plaguing the redshifted $21 \mathrm{~cm}$ line. A crosscorrelation measurement can thus serve as an independent confirmation of the cosmological origin of the measured signals. The use of multiple line tracers would thus be invaluable to validate and enrich our understanding of the EoR, and it will open up a huge discovery space.

\section{References}

Ali-Haïmoud, Y. 2013, Advances in Astronomy, 2013, 2 
Angelakis, E., Kraus, A., Readhead, A. C. S., et al. 2009, A \& A, 501, 801

Basu, K., Hernández-Monteagudo, C., \& Sunyaev, R. A. 2004, A \& A, 416, 447

Bouwens, R. J., Illingworth, G. D., Oesch, P. A., et al. 2014, arXiv1403.4295

Breysse, P. C., Kovetz, E. D., \& Kamionkowski, M. 2014, MNRAS, 443, 3506

Carilli, C. L. 2011, ApJ, 730, L30

Crites, A. T., Bock, J. J., Bradford, C. M., et al. 2014, in Society of Photo-Optical Instrumentation Engineers (SPIE) Conference Series, Vol. 9153, Society of Photo-Optical Instrumentation Engineers (SPIE) Conference Series, 1

De Breuck, C., Williams, R. J., Swinbank, M., et al. 2014, A \& A, 565, A59

Decarli, R., Walter, F., Carilli, C., et al. 2014, ApJ, 782, 78

Gong, Y., Cooray, A., Silva, M., et al. 2012, ApJ, 745, 49

Gong, Y., Cooray, A., Silva, M. B., Santos, M. G., \& Lubin, P. 2011, ApJ, 728, L46

Gong, Y., Silva, M., Cooray, A., \& Santos, M. G. 2014, ApJ, 785, 72

González-López, J., Riechers, D. A., Decarli, R., et al. 2014, ApJ, 784, 99

Hodge, J., Riechers, D. A., Walter, F., et al. 2014, in American Astronomical Society Meeting Abstracts, Vol. 223, American Astronomical Society Meeting Abstracts \#223, \#246.34

Keating, G. K., Bower, G. C., DeBoer, D. R., Heiles, C. E., \& Marrone, D. P. 2014, in American Astronomical Society Meeting Abstracts, Vol. 223, American Astronomical Society Meeting Abstracts \#223, \#133.03

Lidz, A., Furlanetto, S. R., Oh, S. P., et al. 2011, ApJ, 741, 70

Lidz, A., Zahn, O., Furlanetto, S. R., et al. 2009, ApJ, 690, 252

Ota, K., Walter, F., Ohta, K., et al. 2014, ArXiv e-prints

Ouchi, M., Ellis, R., Ono, Y., et al. 2013, ApJ, 778, 102

Pullen, A. R., Chang, T.-C., Doré, O., \& Lidz, A. 2013, ApJ, 768, 15

Pullen, A. R., Doré, O., \& Bock, J. 2014, ApJ, 786, 111

Riechers, D. A., Carilli, C. L., Capak, P. L., et al. 2014, ArXiv e-prints

Righi, M., Hernández-Monteagudo, C., \& Sunyaev, R. A. 2008, A \& A, 489, 489

Robertson, B. E., Furlanetto, S. R., Schneider, E., et al. 2013, ApJ, 768, 71

Salvaterra, R., Ferrara, A., \& Dayal, P. 2011, MNRAS, 414, 847

Sargsyan, L., Lebouteiller, V., Weedman, D., et al. 2012, ApJ, 755, 171

Silva, M. B., santos, M. G., Cooray, A., \& Gong, Y. 2014, ArXiv e-prints

Silva, M. B., Santos, M. G., Gong, Y., Cooray, A., \& Bock, J. 2013, ApJ, 763, 132

Uzgil, B. D., Aguirre, J. E., Bradford, C. M., \& Lidz, A. 2014, ApJ, 793, 116

Visbal, E., \& Loeb, A. 2010, JCAP, 11, 16

Walter, F., Decarli, R., Sargent, M., et al. 2014, ApJ, 782, 79 